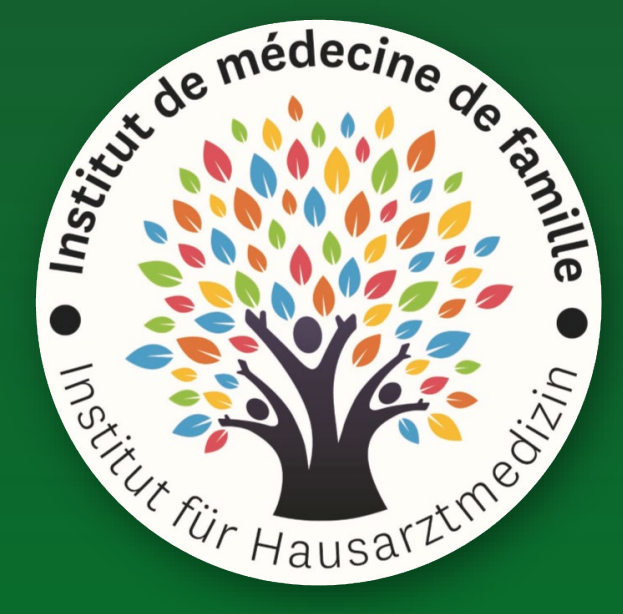

\title{
Enquête sur l'activité des médecins dans le canton de Fribourg, 2020
}

\section{UNI $\boldsymbol{F R}$}




\section{IMPRESSUM}

(C) Institut de médecine de famille (IMF), Université de Fribourg, 2021

Reproduction autorisée, sauf à des fins commerciales, si la référence est mentionnée.

DOI: https://doi.org/10.51363/unifr.srr.2022.001

\section{Analyse des données et rédaction du rapport :}

Angélique Bourquii MSc, Julie Dubois ${ }^{1}$ MA, Arnaud Chiolero ${ }^{2}$ MD PhD, Emmanuelle Lüthi ${ }^{1}$ MSc, PierreYves Rodondi ${ }^{1}, \mathrm{MD}$.

${ }^{1}$ Institut de médecine de famille (IMF), Université de Fribourg

${ }^{2}$ Laboratoire de santé des populations (\#PopHealthLab), Université de Fribourg

\section{Conduite de l'étude :}

$\underline{\mathrm{IMF}}$ : Angélique Bourqui MSc, collaboratrice scientifique ; Julie Dubois MA, collaboratrice scientifique ; Prof. Pierre-Yves Rodondi MD, directeur.

\#PopHealthLab : Prof. Arnaud Chiolero MD PhD, directeur.

Nous remercions l'Observatoire valaisan de la santé (OVS; www.ovs.ch) pour la réalisation de l'enquête, et plus particulièrement David Matthey-Doret, informaticien, et Alexandre Poggio, responsable du secteur Informatique et système d'information.

\section{Partenaire :}

Médecins Fribourg - Ärztinnen und Ärzte Freiburg (MFÄF)

\section{Citation proposée :}

Dubois J, Bourqui A, Lüthi E, Chiolero A, Rodondi PY. Enquête sur l'activité des médecins dans le canton de Fribourg, 2020, Institut de médecine de famille, \#PopHealthLab, Fribourg, décembre 2021.

\section{Disponibilité :}

Institut de médecine de famille : www.unifr.ch/med/imf Langue du texte original : français 


\section{Préface}

Un système de santé avec une forte médecine de famille est moins coûteux et a un impact positif sur la santé de la population. Néanmoins, il y a un risque de pénurie de médecins de famille en Suisse dans les années à venir. La décision du Conseil Fédéral en 2015 d'augmenter le nombre de places pour les étudiant $\cdot$ es en médecine en Suisse était nécessaire, mais la question de savoir combien décideront de s'orienter vers la médecine de famille reste une inconnue. Par ailleurs, il est important de bien connaître dans chaque canton l'offre en médecins de famille, afin d'anticiper les besoins futurs et éviter une pénurie. C'est pour mieux connaître la situation actuelle que l'Institut de médecine de famille (IMF) de l'université de Fribourg a réalisé une enquête auprès des médecins du canton de Fribourg.

Suite au décret du Grand Conseil du canton de Fribourg en 2016, la première volée d'étudiant $\cdot \mathbf{e}$.S a débuté la formation master en médecine en 2019 à l'université de Fribourg. Ce master est unique en Suisse, car il est orienté vers la médecine de famille, tout en délivrant le même diplôme fédéral de médecine que dans les autres universités. Ce master répond à un véritable besoin pour continuer de garantir une haute qualité de soins pour toute la population suisse. II a également pour objectif de contribuer à l'augmentation du nombre de médecins dans d'autres spécialités où un manque est craint.

Une étude menée par l'Institut de médecine de famille de Zurich a montré que plus de $90 \%$ des problèmes de santé peuvent être traités au cabinet du médecin de famille. Une médecine de famille forte, disponible pour la population de toutes les régions de Suisse, est donc un chaînon important du système de santé de notre pays. Pourtant, une enquête menée par la Fédération romande des consommateurs, publiée en novembre 2021, a montré la difficulté à trouver une nouvelle ou un nouveau médecin de famille en Suisse romande. On constate également qu'une part importante des médecins installé $\cdot e \cdot s$ ont plus de 55 ans et qu'ils ont tendance à réduire leur temps de travail hebdomadaire, préférant plus souvent une activité à temps partiel. Tous ces éléments doivent être pris en compte dans le calcul du nombre de médecins à former en médecine de famille. Les données actuelles laissent supposer qu'un effort important est nécessaire pour garantir à l'avenir une densité suffisante de médecins de premier recours dans le canton.

L'institut de médecine de famille (www.unifr.ch/med/imf/fr/) a pour but d'assurer un enseignement de qualité en médecine de famille aux étudiant·ess en médecine de l'université de Fribourg et aussi d'inciter les futur.e.s médecins à ouvrir leur cabinet dans le canton de Fribourg, afin d'assurer une offre adéquate. Toutefois, des données précises manquent, que ce soit notamment sur le nombre de médecins installé·e.s dans le canton, leur âge, leur pourcentage d'activité, ou l'évolution de l'offre, en termes d'augmentation ou diminution d'activité dans les prochaines années.

Nous avons donc réalisé une enquête dont les résultats sont résumés dans ce rapport. Cette enquête a été menée avec le soutien de la Société de médecine du canton de Fribourg (MFÄF), que nous tenons à remercier. Par ailleurs, les données ont été collectées et analysées avec l'étroite collaboration de l'Observatoire valaisan de la santé (OVS; www.ovs.ch) et du Laboratoire de santé des populations (\#PopHealthLab) de l'université de Fribourg, qui ont une grande expérience dans ce type d'analyses.

Les résultats sont intéressants, mais il nous faut plus d'information, notamment pour suivre l'évolution de la situation. Un monitoring cantonal régulier et représentatif est nécessaire, afin de continuer à suivre la situation et chercher les meilleures options pour assurer une couverture médicale adéquate dans chaque district du canton de Fribourg.

Bonne lecture !

Prof. Pierre-Yves Rodondi

Directeur

Institut de médecine de famille

Université de Fribourg 


\section{Résumé}

- L'enquête a été conduite entre le 24 novembre 2020 et le 8 janvier 2021 auprès de l'ensemble des médecins ayant un droit de pratique à titre indépendant (y compris hospitaliers) dans le canton de Fribourg.

- Sur 1252 médecins invité e.s à participer, 228 (22\%) ont rempli le questionnaire. Parmi les participant.e.s, 111 (49\%) sont des médecins de premiers recours (MPR).

- Les principaux résultats sont rapportés dans le tableau ci-dessous. Etant donné le bas taux de réponse à l'enquête, ces résultats doivent être interprétés avec prudence.

\begin{tabular}{|c|c|c|c|}
\hline \multicolumn{2}{|l|}{ Caractéristique } & Total & $\begin{array}{c}\text { Médecins } \\
\text { de } \\
\text { premiers } \\
\text { recours }\end{array}$ \\
\hline \multicolumn{2}{|l|}{ Nombre de participant $\cdot e \cdot s$} & 228 & 111 \\
\hline \multicolumn{2}{|l|}{ Âge médian des participant·e·s } & 54 & 50 \\
\hline \multicolumn{2}{|l|}{ Femmes } & $37 \%$ & $44 \%$ \\
\hline \multicolumn{2}{|c|}{ Demi-journées moyennes d'activité hebdomadaire } & 7.3 & 7.5 \\
\hline \multicolumn{2}{|c|}{ Nombre d'heures moyen d'activité hebdomadaire } & 42.0 & 41.5 \\
\hline \multicolumn{2}{|c|}{ Nombre d'heures moyen d'activité administrative hebdomadaire } & 8.4 & 7.3 \\
\hline \multicolumn{2}{|c|}{ Nombre d'heures par mois consacrées à la formation continue } & $0-10$ & $0-10$ \\
\hline \multirow{4}{*}{ Structure du cabinet } & $\begin{array}{l}\text { Cabinet à deux médecins ou de } \\
\text { groupe }\end{array}$ & $45 \%$ & $69 \%$ \\
\hline & Cabinet individuel & $33 \%$ & $31 \%$ \\
\hline & $\begin{array}{l}\text { Médecin dans un établissement } \\
\text { hospitalier }\end{array}$ & $15 \%$ & n.a \\
\hline & Autre & $7 \%$ & n.a \\
\hline \multicolumn{2}{|c|}{ Projet de diminution du taux d'activité dans les 5 ans } & $35 \%$ & $32 \%$ \\
\hline \multirow{3}{*}{$\begin{array}{l}\text { Accepte un nouveau ou une nouvelle } \\
\text { patient } \cdot e^{*}\end{array}$} & Dans un délai de 5 jours & $36 \%$ & $50 \%$ \\
\hline & Dans un délai d'un mois & $64 \%$ & $67 \%$ \\
\hline & N'en accepte pas & $14 \%$ & $17 \%$ \\
\hline \multicolumn{2}{|c|}{$\begin{array}{l}\text { Offre en médecine de premier recours dans } 5 \text { ans estimée insuffisante par les } \\
\text { médecins participant à l'enquête }\end{array}$} & $56 \%$ & $58 \%$ \\
\hline \multicolumn{2}{|l|}{ Participation à la garde } & $58 \%$ & $67 \%$ \\
\hline
\end{tabular}

*La proportion de médecins qui acceptent un nouveau ou une nouvelle patient·e dans un délai d'un mois inclut ceux qui acceptent un nouveau ou une nouvelle patient·e dans un délai de moins de 5 jours.

- L'âge médian de l'ensemble des participant·e-s est de 54 ans (48 ans chez les femmes et 57 ans chez les hommes). L'âge médian est de 50 ans chez les MPR ( 45 ans chez les femmes et 58 ans chez les hommes). La majorité des participant·e.s sont des hommes, y compris dans le sous-groupe des MPR.

- La durée moyenne d'activité hebdomadaire s'élève à 42.0 heures et 8.4 heures ( $20 \%$ du temps) sont consacrées aux tâches administratives. Les MPR travaillent en moyenne 41.5 heures par semaine, dont 7.3 heures ( $18 \%$ du temps) pour des tâches administratives.

- Parmi le total des participant·e.s, un peu moins de la moitié pratique dans un cabinet à deux médecins ou de groupe. Ce chiffre s'élève à un peu plus de deux tiers dans le sous-groupe des MPR.

- Près d'un tiers des participant·e.s envisage de diminuer son taux d'activité dans les 5 ans, y compris dans le sous-groupe des MPR (en majorité d'une à deux demi-journées par semaine). 
- $14 \%$ des participant $\cdot e \cdot s(17 \%$ des MPR) n'accepte pas de nouvelle patiente ou de nouveau patient.

- Plus de la moitié des participant·e·s considère que l'offre médicale pour la médecine de premier recours sera insuffisante dans 5 ans.

- Parmi le total des participant·e·s, plus de la moitié participe à la garde. Ce chiffre s'élève à deux tiers dans le sous-groupe des MPR.

\section{Zusammenfassung}

- Die Umfrage wurde zwischen dem 24. November 2020 und dem 8. Januar 2021 bei allen Ärzten und Ärztinnen mit Bewilligung zur selbstständigen Berufsausübung (einschliesslich Spitalärzte) im Kanton Freiburg durchgeführt.

- Von 1252 Ärzten und Ärztinnen, die zur Teilnahme eingeladen wurden, füllten 228 (22\%) den Fragebogen aus. Von den Teilnehmenden waren 111 (49\%) Hausärzte, Hausärztinnen.

- Die wichtigsten Ergebnisse sind in der nachstehenden Tabelle zusammengefasst. Angesichts der niedrigen Rücklaufquote der Umfrage sollten diese Ergebnisse mit Vorsicht interpretiert werden.

\begin{tabular}{|c|c|c|c|}
\hline \multicolumn{2}{|l|}{ Themen } & Total & Hausärzte \\
\hline \multicolumn{2}{|l|}{ Anzahl der Teilnehmer/innen } & 228 & 111 \\
\hline \multicolumn{2}{|l|}{ Medianalter der Teilnehmer/innen } & 54 & 50 \\
\hline \multicolumn{2}{|l|}{ Frauen } & $37 \%$ & $44 \%$ \\
\hline \multicolumn{2}{|c|}{ Durchschnittliche Halbtage der wöchentlichen Aktivität } & 7.3 & 7.5 \\
\hline \multicolumn{2}{|c|}{ Durchschnittliche Stundenzahl der wöchentlichen Aktivität } & 42.0 & 41.5 \\
\hline \multicolumn{2}{|c|}{ Durchschnittliche Anzahl an Stunden pro Woche für administrative Tätigkeiten } & 8.4 & 7.3 \\
\hline \multicolumn{2}{|c|}{ Anzahl Stunden pro Monat, die für die Weiterbildung aufgewendet werden } & $0-10$ & $0-10$ \\
\hline \multirow{4}{*}{ Struktur der Praxis } & $\begin{array}{l}\text { Praxis mit zwei Ärzten, Ärztinnen } \\
\text { oder Gruppenpraxis }\end{array}$ & $45 \%$ & $69 \%$ \\
\hline & Einzelpraxis & $33 \%$ & $31 \%$ \\
\hline & Arzt/Ärztin in einem Spital & $15 \%$ & n.a \\
\hline & Andere & $7 \%$ & n.a \\
\hline \multicolumn{2}{|c|}{ Projekt zur Reduktion des Beschäftigungsgrades in den nächsten fünf Jahren } & $35 \%$ & $32 \%$ \\
\hline \multirow{3}{*}{$\begin{array}{l}\text { Nimmt einen neuen Patienten, eine neue } \\
\text { Patientin auf* }\end{array}$} & Innerhalb von 5 Tagen & $36 \%$ & $50 \%$ \\
\hline & Innerhalb von einem Monat & $64 \%$ & $67 \%$ \\
\hline & $\begin{array}{l}\text { Nimmt keine neuen } \\
\text { Patienten/innen auf }\end{array}$ & $14 \%$ & $17 \%$ \\
\hline \multicolumn{2}{|c|}{$\begin{array}{l}\text { Anteil der an der Umfrage teilnehmenden Ärzte/Ärztinnen, die das Angebot an } \\
\text { Hausarztmedizin in den nächsten } 5 \text { Jahren als unzureichend einschätzen }\end{array}$} & $56 \%$ & $58 \%$ \\
\hline \multicolumn{2}{|l|}{ Teilnahme am Bereitschaftsdienst } & $58 \%$ & $67 \%$ \\
\hline
\end{tabular}

* Der Anteil der Ärzte, die einen neuen Patienten/eine neue Patientin innerhalb eines Monats annehmen, schließt diejenigen ein, die einen neuen Patienten/eine neue Patientin innerhalb von weniger als fünf Tagen annehmen.

- Das Medianalter aller Teilnehmenden beträgt 54 Jahre (48 Jahre bei den Frauen und 57 Jahre bei den Männern). Das Medianalter der Hausärzte, Hausärztinnen beträgt 50 Jahre (45 Jahre bei den Frauen und 58 Jahre bei den Männern). Die Mehrheit der Teilnehmenden sind Männer, auch in der Untergruppe der Hausärzte und Hausärztinnen.

- Die durchschnittliche wöchentliche Aktivitätsdauer beträgt 42 Stunden und 8.4 Stunden $(20 \%$ der Zeit) werden für administrative Aufgaben aufgewendet. Die Hausärzte, Hausärztinnen arbeiten durchschnittlich 41.5 Stunden pro Woche, davon entfallen 7.3 Stunden (18\% der Zeit) auf administrative Aufgaben. 
- Von allen Teilnehmenden praktiziert etwas weniger als die Hälfte in einer Praxis mit zwei Ärzten, Ärztinnen oder in einer Gemeinschaftspraxis. In der Untergruppe der Hausärztinnen und Hausärzte sind es etwas mehr als zwei Drittel.

- Von allen Teilnehmenden plant ein Drittel, den Beschäftigungsgrad innerhalb von 5 Jahren zu reduzieren, auch in der Untergruppe der Hausärztinnen und Hausärzte (mehrheitlich um einen bis zwei halbe Tage pro Woche).

- $14 \%$ der Teilnehmenden (17\% der Hausärzte, Hausärztinnen) nehmen keine neuen Patient_innen mehr an.

- Mehr als die Hälfte der Teilnehmenden ist der Ansicht, dass das medizinische Angebot für die Hausarztmedizin in fünf Jahren unzureichend sein wird.

- Von der Gesamtzahl der Teilnehmenden nimmt mehr als die Hälfte am Bereitschaftsdienst teil. In der Untergruppe der Hausärztinnen und Hausärzte beträgt diese Zahl zwei Drittel. 


\section{Contexte et buts de l'enquête}

Selon les données de l'OBSAN de 2017 (OBSAN, 2017), le canton de Fribourg figure parmi les cantons avec la plus faible densité de médecins en cabinet privé (spécialistes et de premier recours) de Suisse. Des données plus précises sont nécessaires, pour mieux comprendre cette situation et obtenir des projections pour le futur. Dès lors, l'Institut de médecine de famille (IMF) de l'Université de Fribourg a décidé de réaliser une enquête sur l'activité des médecins ayant une autorisation de pratique dans le canton de Fribourg. Cette enquête a été réalisée en partenariat avec le Laboratoire de santé des populations (\#PopHealthLab) et Médecins Fribourg (MFÄF).

\section{Méthode}

Pour la réalisation de cette enquête, l'IMF a mandaté l'Observatoire valaisan de la santé (OVS ; www.ovs.ch). L'enquête a été conduite entre le 24 novembre 2020 et le 8 janvier 2021.

\section{Population cible et questionnaire}

L'ensemble des médecins ayant un droit de pratique à titre indépendant (y compris hospitaliers) dans le canton de Fribourg a reçu une invitation à participer à l'enquête. La liste des médecins éligibles a été établie et transmise par MFÄF.

Le questionnaire utilisé pour cette enquête (Annexe 1) reprend pour l'essentiel celui qui avait été élaboré par l'OVS dans le cadre de l'enquête sur l'activité des médecins en Valais, datée de 2018 (Enquête OVS, 2018). II a été complété par un set de questions portant notamment sur le nombre d'heures hebdomadaires consacrées au travail administratif et à la formation continue.

Les médecins ont été convié·e·s à participer à l'enquête par l'OVS, au travers d'un e-mail contenant un lien unique (personnel) leur permettant d'accéder au questionnaire en ligne. En cas de non-réponse, un $1^{\mathrm{er}}$ et un $2^{\mathrm{e}}$ rappels ont été envoyés par e-mail environ 10 jours après le précédent e-mail. Un dernier rappel a été effectué 2 jours avant la clôture du questionnaire. Certaines adresses e-mail n'étant pas valides, et afin d'augmenter le taux de réponse, les personnes concernées ont reçu un courrier postal unique le 8 décembre 2020, sans rappel.

L'échantillon initial comportait 1'252 adresses e-mail. Les doublons $(n=4)$, les adresses introuvables $(n=85)$ ainsi que les médecins à la retraite ou ne travaillant plus dans le canton de Fribourg $(n=106)$ ont été retirés de l'échantillon initial. Sur les 1'057 médecins éligibles et invité·e·s, 829 n'ont pas complété le questionnaire. L'échantillon final compte par conséquent 228 médecins qui ont répondu à l'entier du questionnaire (Figure 1). Le taux de participation est de $22 \%$.

Figure 1. Médecins ayant participé à l'enquête par questionnaire.

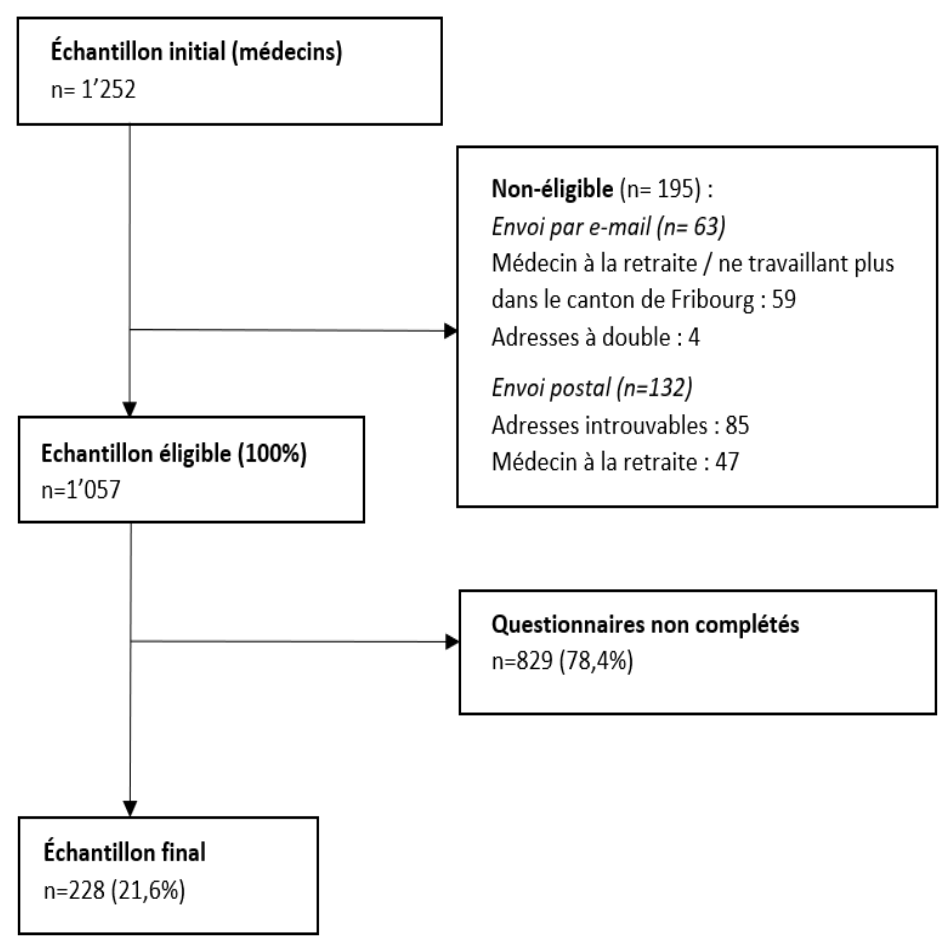




\section{Analyse des données}

Le traitement des données a été réalisé par I'OVS et leur analyse par l'IMF. Le traitement des données comprenait notamment la préparation d'une base de données anonymisée, ainsi que la production de tableaux et de graphiques selon les demandes de l'IMF. Les tableaux et graphiques ainsi que la base de données anonymisée complète ont ensuite été livrés à l'IMF. L'OVS ne garde pas de copie de cette base de données.

\section{Définition des médecins de premier recours (MPR)}

Dans ce rapport, les médecins sont considéré·e.s comme ayant une activité de premier recours s'ils ou elles ont répondu pratiquer de la médecine de premier recours, ne sont pas médecin cadre dans un hôpital, ont une adresse de pratique en cabinet médical et sont titulaires d'un titre de spécialiste de médecine interne générale, pédiatrie, médecin praticien-ne ou d'un titre de spécialiste apparenté. Quarante-neuf pourcent des répondant·ess sont médecins de premier recours et des analyses spécifiques ont été effectuées pour cette population. 


\section{Résultats détaillés}

\begin{tabular}{|c|c|c|}
\hline $\mathrm{Nr}$ & Descriptif & Lien \\
\hline 1 & Caractéristiques des participant $\cdot \mathrm{e} \cdot \mathrm{s}$ & Caractéristiques des participant.e.s \\
\hline 2 & Principale activité & $\underline{\text { Principale activité }}$ \\
\hline 3 & $\begin{array}{l}\text { Nombre d'heures et de demi-journées selon } \\
\text { l'activité totale, principale et administrative }\end{array}$ & $\begin{array}{l}\text { Nombre d'heures et de demi-journées } \\
\text { selon l'activité totale, principale et } \\
\text { administrative }\end{array}$ \\
\hline 4 & Modifications du taux d'activité envisagées & $\begin{array}{l}\text { Modifications du taux d'activité } \\
\text { envisagées }\end{array}$ \\
\hline 5 & Participation à la garde & Participation à la garde \\
\hline 6 & $\begin{array}{l}\text { Temps d'attente pour une consultation pour un } \cdot e \\
\text { nouveau ou nouvelle patient } e\end{array}$ & $\begin{array}{l}\text { Temps d'attente pour une consultation } \\
\text { pour un'e nouveau ou nouvelle patient } \cdot \text { e }\end{array}$ \\
\hline 7 & Evaluation de l'offre médicale & Evaluation de l'offre médicale \\
\hline 8 & Discussion des résultats et conclusion & Discussion des résultats et conclusion \\
\hline 9 & Annexe 1: Questionnaire & Annexe 1: Questionnaire \\
\hline
\end{tabular}




\section{Caractéristiques des participant·e·s}

Figure 1.1a. Répartition de l'ensemble des médecins en fonction de la classe d'âge.

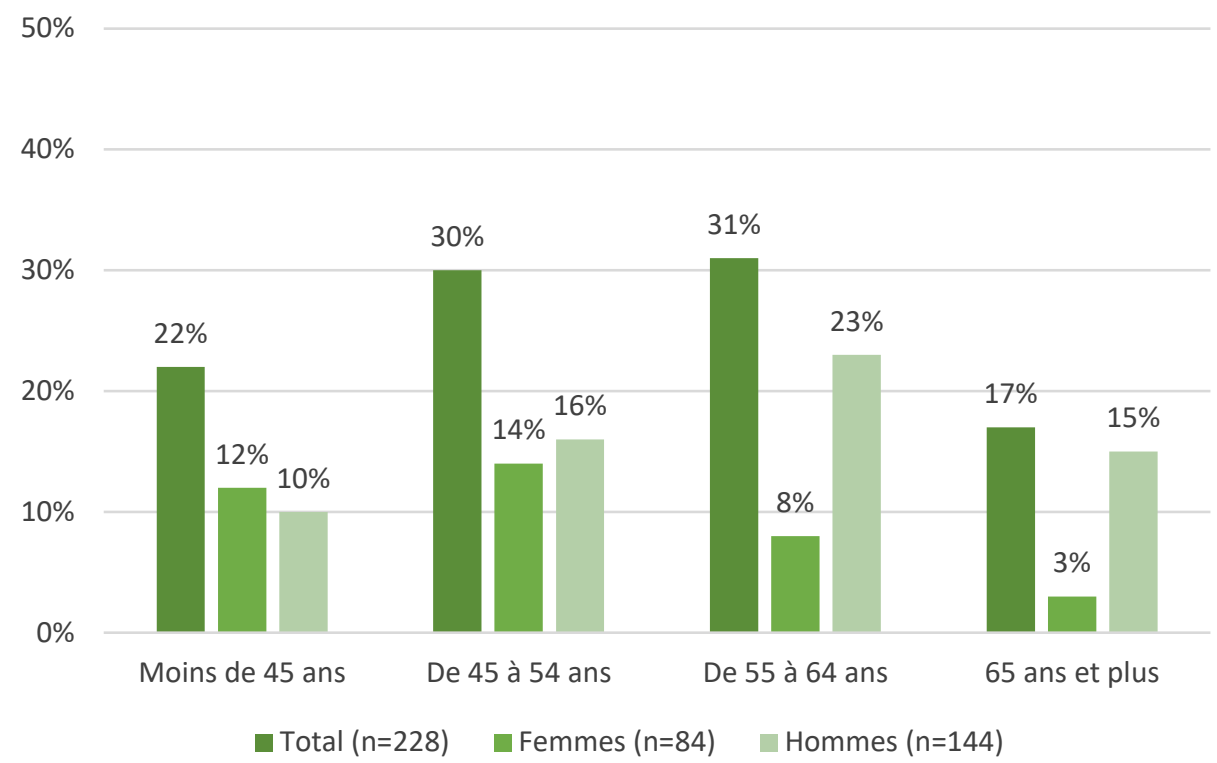

Figure 1.1b. Répartition des médecins de premier recours en fonction de la classe d'âge.

$50 \%$

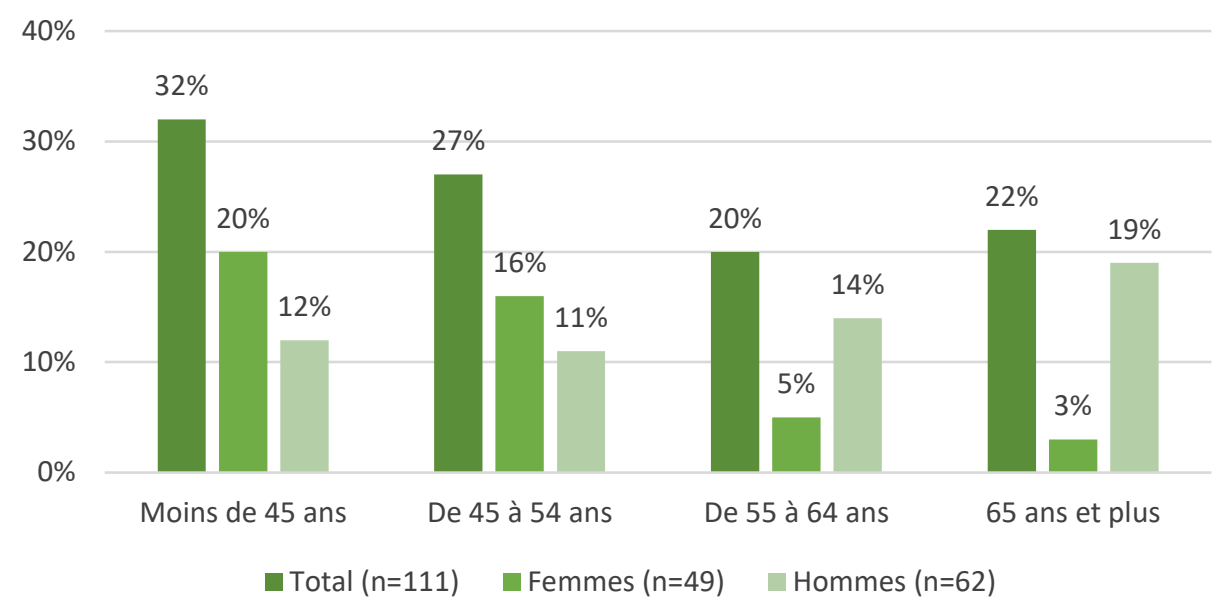

Les femmes sont plus nombreuses dans la catégorie d'âge de 45 à 54 ans alors que les hommes sont plus nombreux dans la catégorie d'âge de 55 à 64 ans. Pour le sous-groupe des MPR, les femmes sont plus nombreuses dans la catégorie d'âge de moins de 45 ans et les hommes dans la catégorie de 65 ans et plus. Lorsque l'on considère l'ensemble des participant·e·s, l'âge médian des femmes est de 48 ans et celui des hommes de 57 ans. Chez les MPR, l'âge médian est de 45 ans chez les femmes et de 58 ans chez les hommes. 
Tableau 1.1 Nombre de participant $\cdot e \cdot s$ par districts.

\begin{tabular}{lcc}
\hline Districts & $\mathbf{N}_{\text {total }}(\%)$ & $\mathbf{N}_{\text {MPR }}(\%)$ \\
\hline Glâne & $13(6)$ & $5(5)$ \\
\hline Gruyère & $45(20)$ & $22(20)$ \\
\hline Veveyse & $7(3)$ & $7(6)$ \\
\hline Broye & $12(5)$ & $11(10)$ \\
\hline Sarine & $102(45)$ & $32(29)$ \\
\hline Singine & $32(14)$ & $23(21)$ \\
\hline Lac & $17(8)$ & $11(10)$ \\
\hline Total & $\mathbf{2 2 8 ( 1 0 0 )}$ & $\mathbf{1 1 1 ( 1 0 0 )}$ \\
\hline
\end{tabular}

Près de la moitié des participant $\cdot e \cdot s$ exerce dans le district de la Sarine. 


\section{Principale activité}

Tableau 2.1 Nombre de participant·e·s selon leur titre de spécialiste (selon la liste des spécialités ISFM ou reconnaissance MEBEKO).

\begin{tabular}{|c|c|}
\hline Titre de spécialiste & $\mathbf{N}_{\text {total }}(\%)$ \\
\hline Médecine interne générale & $108(47)$ \\
\hline Autre & $29(13)$ \\
\hline Psychiatrie et psychothérapie & $22(10)$ \\
\hline Pédiatrie & $16(7)$ \\
\hline Chirurgie orthopédique et traumatologie de l'appareil locomoteur & $13(6)$ \\
\hline Anesthésiologie & $10(4)$ \\
\hline Médecin praticien·ne & $10(4)$ \\
\hline Chirurgie & $8(4)$ \\
\hline Gynécologie et obstétrique & $7(3)$ \\
\hline Psychiatrie et psychothérapie d'enfants et d'adolescent·e.s & $5(2)$ \\
\hline Cardiologie & $4(2)$ \\
\hline Ophtalmologie & $4(2)$ \\
\hline Rhumatologie & $4(2)$ \\
\hline Allergologie et immunologie clinique & $3(1)$ \\
\hline Oto-rhino-laryngologie & $3(1)$ \\
\hline Pathologie & $3(1)$ \\
\hline Radiologie & $3(1)$ \\
\hline Dermatologie et vénéréologie & $2(1)$ \\
\hline Endocrinologie / diabétologie & $2(1)$ \\
\hline Gastroentérologie & $2(1)$ \\
\hline Infectiologie & $2(1)$ \\
\hline Médecine du travail & $2(1)$ \\
\hline Médecine tropicale et médecine des voyages & $2(1)$ \\
\hline Pneumologie & $2(1)$ \\
\hline Soins intensifs & $2(1)$ \\
\hline Chirurgie de la main & $1(<1)$ \\
\hline Chirurgie pédiatrique & $1(<1)$ \\
\hline Chirurgie plastique, reconstructive et esthétique & $1(<1)$ \\
\hline Génétique médicale & $1(<1)$ \\
\hline Gériatrie & $1(<1)$ \\
\hline Médecine physique et réadaptation & $1(<1)$ \\
\hline Neurochirurgie & $1(<1)$ \\
\hline Neurologie & $1(<1)$ \\
\hline Oncologie médicale & $1(<1)$ \\
\hline
\end{tabular}




\begin{tabular}{lc}
\hline Angiologie & $0(0)$ \\
\hline Chirurgie cardiaque et vasculaire thoracique & $0(0)$ \\
\hline Chirurgie orale et maxillo-faciale & $0(0)$ \\
\hline Chirurgie thoracique & $0(0)$ \\
\hline Chirurgie vasculaire & $0(0)$ \\
\hline Hématologie & $0(0)$ \\
\hline Médecine légale & $0(0)$ \\
\hline Médecine nucléaire & $0(0)$ \\
\hline Médecine pharmaceutique & $0(0)$ \\
\hline Néphrologie & $0(0)$ \\
\hline Neuropathologie & $0(0)$ \\
\hline Pharmacologie et toxicologie cliniques & $0(0)$ \\
\hline Prévention et santé publique & $0(0)$ \\
\hline Radio-oncologie/radiothérapie & $0(0)$ \\
\hline Urologie & $0(0)$ \\
\hline Total des répondant·e·s & $\mathbf{2 2 8}(\mathbf{1 0 0})$ \\
\hline
\end{tabular}

Près de la moitié des participant·e·s a un titre de spécialiste en médecine générale interne.

Tableau 2.2 Nombre de participant·e·s selon leur principale activité - principale spécialisation exercée.

\begin{tabular}{ll}
\hline Principale activité - principale spécialisation exercée & $\mathbf{N}_{\text {total }}(\%)$ \\
\hline Médecine interne générale & $95(42)$ \\
\hline Psychiatrie et psychothérapie & $22(10)$ \\
\hline Pédiatrie & $16(7)$ \\
\hline Chirurgie orthopédique et traumatologie de l'appareil locomoteur & $12(5)$ \\
\hline Autre & $10(4)$ \\
\hline Anesthésiologie & $7(3)$ \\
\hline Chirurgie & $7(3)$ \\
\hline Gynécologie et obstétrique & $7(3)$ \\
\hline Médecin praticien & $7(3)$ \\
\hline Psychiatrie et psychothérapie d'enfants et d'adolescents & $5(2)$ \\
\hline Ophtalmologie & $4(2)$ \\
\hline Oto-rhino-laryngologie & $4(2)$ \\
\hline Rhumatologie & $4(2)$ \\
\hline Cardiologie & $3(1)$ \\
\hline Pathologie & $3(1)$ \\
\hline Radiologie & $3(1)$ \\
\hline Allergologie et immunologie clinique & $2(1)$ \\
\hline Chirurgie plastique, reconstructive et esthétique & $2(1)$ \\
\hline
\end{tabular}




\begin{tabular}{lc}
\hline Dermatologie et vénéréologie & $2(1)$ \\
\hline Endocrinologie-diabétologie & $2(1)$ \\
\hline Gastroentérologie & $2(1)$ \\
\hline Médecine du travail & $2(1)$ \\
\hline Génétique médicale & $1(<1)$ \\
\hline Maladies infectieuses (Infectiologie) & $1(<1)$ \\
\hline Médecine physique et réadaptation & $1(<1)$ \\
\hline Neurochirurgie & $1(<1)$ \\
\hline Neurologie & $1(<1)$ \\
\hline Oncologie médicale & $1(<1)$ \\
\hline Pneumologie & $1(<1)$ \\
\hline Autres & $0(0)$ \\
\hline Total & $228(100)$ \\
\hline
\end{tabular}

Un peu moins de la moitié des participant·e·s a comme activité principale la médecine interne générale.

Tableau 2.3 Nombre de participant $\cdot e \cdot s$ selon la structure de leur cabinet.

\begin{tabular}{lcc}
\hline Structure du cabinet & $\mathbf{N}_{\text {total }}(\%)$ & $\mathbf{N}_{\text {MPR }}(\%)$ \\
\hline Cabinet à deux médecins ou de groupe & $103(45)$ & $77(69)$ \\
\hline Cabinet individuel & $75(33)$ & $34(31)$ \\
\hline Médecin dans un établissement hospitalier & $35(15)$ & n.a \\
\hline Autre & $15(7)$ & n.a \\
\hline Total & $\mathbf{2 2 8 ( 1 0 0 )}$ & $\mathbf{1 1 1 ( 1 0 0 )}$ \\
\hline
\end{tabular}

Parmi le total des participant $\cdot \mathrm{e} \cdot \mathrm{s}$, un peu moins de la moitié pratique dans un cabinet à deux médecins ou de groupe et un tiers dans un cabinet individuel. Dans le sous-groupe des MPR, les deux tiers pratiquent dans un cabinet à deux médecins ou de groupe. 


\section{Nombre d'heures et de demi-journées selon l'activité totale, principale et administrative}

Figure 3.1 Répartition des médecins et des médecins de premier recours en fonction du nombre d'heures de travail par semaine.

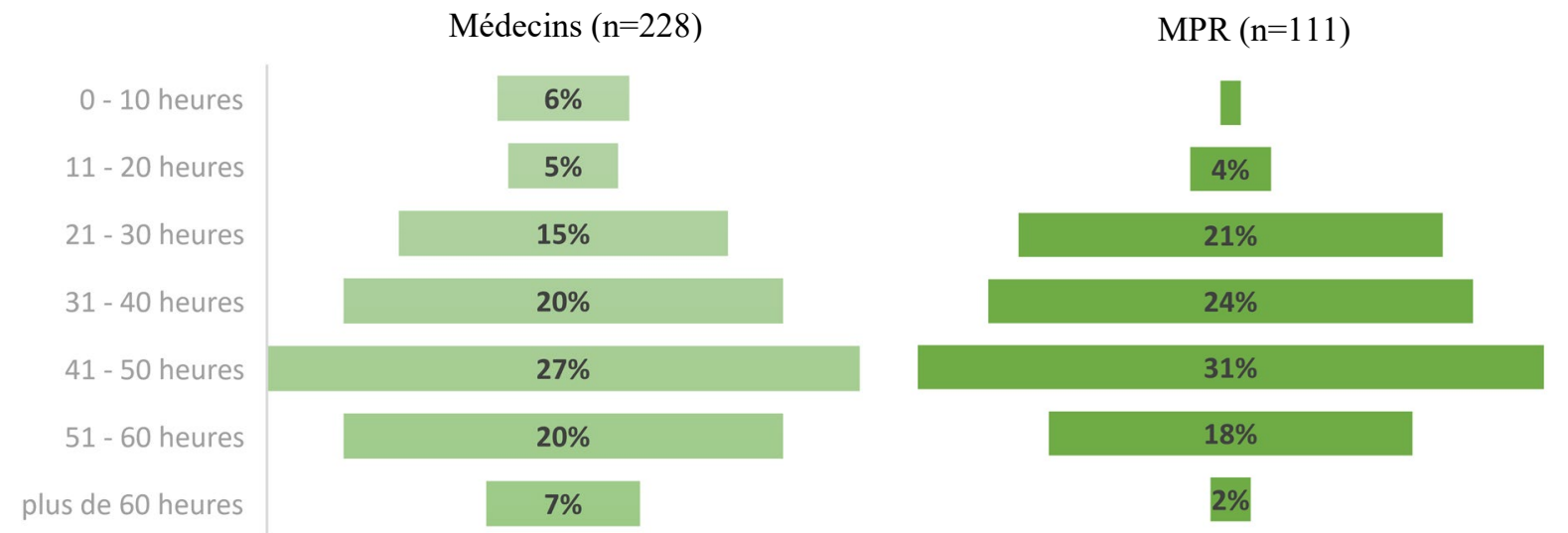

Dans les deux groupes de participant·e.s, la majorité consacre entre 41-50 heures hebdomadaires à la pratique de son métier. En moyenne, les participant·e's consacrent 42 heures hebdomadaires à la pratique de leur métier (41 heures 30 dans le sous-groupe des MPR).

Tableau 3.1 Nombre d'heures par semaine en moyenne consacrées au travail administratif (gestion de l'activité, comptabilité, rapports d'assurance, lettres, etc).

\begin{tabular}{lcc}
\hline Nombre d'heures & $\mathbf{N}_{\text {total }}(\%)$ & $\mathbf{N}_{\text {MPR }}(\%)$ \\
\hline $0-10$ heures & $183(80)$ & $93(84)$ \\
\hline $11-20$ heures & $34(15)$ & $16(14)$ \\
\hline $21-30$ heures & $3(1)$ & $2(2)$ \\
\hline $31-40$ heures & $6(3)$ & $0(0)$ \\
\hline $41-50$ heures & $1(<1)$ & $0(0)$ \\
\hline $51-60$ heures & $0(0)$ & $0(0)$ \\
\hline Plus 60 heures & $1(<1)$ & $0(0)$ \\
\hline Total & $\mathbf{2 2 8 ( 1 0 0 )}$ & $\mathbf{1 1 1 ( 1 0 0 )}$ \\
\hline
\end{tabular}

Dans les deux groupes de participant·e·s, la majorité consacre entre 0-10 heures hebdomadaires au travail administratif. En moyenne, les participant.e.s dans leur ensemble consacrent 8.4 heures hebdomadaires au travail administratif (20\% des heures en moyenne). Les MPR y consacrent en moyenne 7.3 heures ( $18 \%$ des heures en moyenne). 
Tableau 3.2 Nombre de demi-journée(s) d'activité totale lors d'une semaine habituelle.

\begin{tabular}{lcc}
\hline Demi-journées & $\mathbf{N}_{\text {total }}(\%)$ & $\mathbf{N}_{\text {MPR }}(\%)$ \\
\hline 0 demi-journée & $11(5)$ & $0(0)$ \\
\hline 1 demi-journée & $4(2)$ & $2(2)$ \\
\hline 2 demi-journées & $6(3)$ & $0(0)$ \\
\hline 3 demi-journées & $4(2)$ & $0(0)$ \\
\hline 4 demi-journées & $4(2)$ & $4(4)$ \\
\hline 5 demi-journées & $20(9)$ & $10(9)$ \\
\hline 6 demi-journées & $35(1)$ & $24(22)$ \\
\hline 7 demi-journées & $22(10)$ & $15(14)$ \\
\hline 8 demi-journées & $34(15)$ & $16(14)$ \\
\hline 9 demi-journées & $30(13)$ & $18(16)$ \\
\hline 10 demi-journées & $42(18)$ & $17(15)$ \\
\hline 11 demi-journées & $12(5)$ & $3(3)$ \\
\hline 12 demi-journées & $3(1)$ & $1(1)$ \\
\hline 13 demi-journées & $0(0)$ & $0(0)$ \\
\hline 14 demi-journées & $1(0)$ & $1(1)$ \\
\hline Total & $\mathbf{2 2 8 ( 1 0 0 )}$ & $\mathbf{1 1 1}(\mathbf{1 0 0})$ \\
\hline Demi-journées moyennes & $\mathbf{7 . 3}$ & \\
\hline d'activité & & 7.5 \\
\hline & 7 &
\end{tabular}

La majorité des participant·e.s pratique entre 7 et 10 demi-journées par semaine (y compris le travail administratif), avec une moyenne de 7.3 demi-journées pour l'ensemble des participant·e.s. La majorité des MPR pratique entre 6 et 10 demi-journées par semaine (y compris le travail administratif), avec une moyenne de 7.5 demi-journées.

Tableau 3.3 Nombre d'heures par mois en moyenne consacrées à la formation continue.

\begin{tabular}{lcc}
\hline Nombre d'heures & $\mathbf{N}_{\text {total }}(\%)$ & $\mathbf{N}_{\text {MPR }}(\%)$ \\
\hline $0-10$ heures & $212(93)$ & $107(96)$ \\
\hline $11-20$ heures & $13(6)$ & $3(3)$ \\
\hline $21-30$ heures & $2(1)$ & $0(0)$ \\
\hline $31-40$ heures & $0(0)$ & $0(0)$ \\
\hline $41-50$ heures & $0(0)$ & $0(0)$ \\
\hline $51-60$ heures & $0(0)$ & $0(0)$ \\
\hline plus de 60 heures & $1(<0)$ & $1(1)$ \\
\hline Total & $\mathbf{2 2 8 ( 1 0 0 )}$ & $\mathbf{1 1 1 ( 1 0 0 )}$ \\
\hline
\end{tabular}

La majorité des participant $\cdot e \cdot s$ consacre entre $0-10$ heures par mois à la formation continue, y compris dans le sous-groupe des MPR. 


\section{Modifications envisagées du taux d'activité}

Tableau 4.1 Projet de modification du taux d'activité dans les 5 ans.

\begin{tabular}{lcc}
\hline $\begin{array}{l}\text { Projet de modification du taux } \\
\text { d'activité }\end{array}$ & $\mathbf{N}_{\text {total }}(\%)$ & $\mathbf{N}_{\text {MPR }}(\%)$ \\
\hline Oui, augmentation & $12(5)$ & $4(4)$ \\
\hline Oui, diminution & $79(35)$ & $36(32)$ \\
\hline Non & $137(60)$ & $71(64)$ \\
\hline Total & $\mathbf{2 2 8 ( 1 0 0 )}$ & $\mathbf{1 1 1 ( 1 0 0 )}$ \\
\hline
\end{tabular}

Parmi les participant·e.s, un tiers envisage de diminuer son taux d'activité dans les 5 ans, y compris dans le sous-groupe des MPR.

Tableau 4.2 Projet de diminution du taux d'activité dans les 5 ans.

\begin{tabular}{lcc}
\hline Nombre de demi-journées & $\mathbf{N}_{\text {total }}(\%)$ & $\mathbf{N}_{\text {MPR }}(\%)$ \\
\hline 1 à 2 demi-journées en moins & $53(67)$ & $22(61)$ \\
\hline 3 à 4 demi-journées en moins & $15(19)$ & $6(17)$ \\
\hline 5 demi-journées et plus en moins & $11(14)$ & $8(22)$ \\
\hline Total & $\mathbf{7 9}(100)$ & $\mathbf{3 6}(100)$ \\
\hline
\end{tabular}

Parmi l'ensemble des participant·ess qui prévoient de diminuer leur taux d'activité dans les 5 ans, les deux tiers environ envisagent de diminuer de 1 à 2 demi-journées. Les mêmes résultats sont observés pour le sous-groupe des MPR.

Tableau 4.2.1 Projet de diminution du taux d'activité dans les 5 ans par classe d'âge (uniquement MPR).

\begin{tabular}{lccc}
\hline Âge & $\begin{array}{c}\mathbf{1} \text { à } \mathbf{2} \text { demi-journées } \\
\text { en moins }\end{array}$ & $\begin{array}{c}\text { 3 à } \mathbf{4} \text { demi-journées } \\
\text { en moins }\end{array}$ & $\begin{array}{c}\mathbf{5} \text { demi-journées et plus } \\
\text { en moins }\end{array}$ \\
\hline Moins de 45 ans & $\mathbf{N}_{\text {MPR } \%}$ & $\mathbf{N}_{\text {MPR } \%}$ & $\mathbf{N}_{\text {MPR }}$ (\%) \\
\hline De 45 à 54 ans & $4(11)$ & $0(0)$ & $0(0)$ \\
\hline De 55 à 64 ans & $3(8)$ & $0(0)$ & $0(0)$ \\
\hline 65 ans et plus & $7(20)$ & $2(6)$ & $3(8)$ \\
\hline Total & $8(22)$ & $4(11)$ & $5(14)$ \\
\hline
\end{tabular}

Parmi les MPR qui envisagent de diminuer leur taux d'activité dans les 5 ans $(n=36), 47 \%$ ont 65 ans et plus.

Tableau 4.3 Projet d'augmentation du taux d'activité dans les 5 ans.

\begin{tabular}{lcc}
\hline Nombre de demi-journées & $\mathbf{N}_{\text {total }}(\%)$ & $\mathbf{N}_{\text {MPR }}(\%)$ \\
\hline 1 à 2 demi-journées en plus & $8(67)$ & $4(100)$ \\
\hline 3 à 4 demi-journées en plus & $3(25)$ & $0(0)$ \\
\hline 5 demi-journées et plus en plus & $1(8)$ & $0(0)$ \\
\hline Total & $\mathbf{1 2 ( 1 0 0 )}$ & $\mathbf{4 ( 1 0 0 )}$ \\
\hline
\end{tabular}


Parmi les participant·e.s qui prévoient d'augmenter leur taux d'activité dans les 5 ans, les deux tiers envisagent d'augmenter de 1 à 2 demi-journées. La totalité du sous-groupe des MPR prévoit d'augmenter de 1 à 2 demi-journées. 


\section{Participation à la garde}

Tableau 5.1 Nombre de médecins participant à la garde.

\begin{tabular}{lcc}
\hline Participation à la garde & $\mathbf{N}_{\text {total }}(\%)$ & $\mathbf{N}_{\text {MPR }}(\%)$ \\
\hline Oui & $133(58)$ & $74(67)$ \\
\hline Non & $95(42)$ & $37(33)$ \\
\hline Total & $\mathbf{2 2 8 ( 1 0 0 )}$ & $\mathbf{1 1 1 ( 1 0 0 )}$ \\
\hline
\end{tabular}

Plus de la moitié de l'ensemble des participant·e.s participe à la garde. Deux tiers du sous-groupe des MPR y participent.

Tableau 5.2 Nombre de médecins participant à la garde par nombre de jours de garde effectués par année.

\begin{tabular}{lcc}
\hline Nombre de jours de garde & $\mathbf{N}_{\text {total }}(\%)$ & $\mathbf{N}_{\text {MPR }}(\%)$ \\
\hline 2 - 5 jours & $16(12)$ & $13(18)$ \\
\hline 6 jours à 1 mois & $92(69)$ & $58(78)$ \\
\hline Entre 1 mois et 6 mois & $23(17)$ & $3(4)$ \\
\hline Entre 6 mois et 1 an & $2(2)$ & $0(0)$ \\
\hline Total & $\mathbf{1 3 3 ( 1 0 0 )}$ & $\mathbf{7 4 ( 1 0 0 )}$ \\
\hline
\end{tabular}

La majorité des participant·e·s participe à la garde 6 jours à 1 mois par année, y compris dans le sousgroupe des MPR. 


\section{Temps d'attente pour une consultation pour un nouveau ou une nouvelle patient·e}

Figure 6.1 Temps d'attente pour l'obtention d'une consultation pour un nouveau ou une nouvelle patient.e.

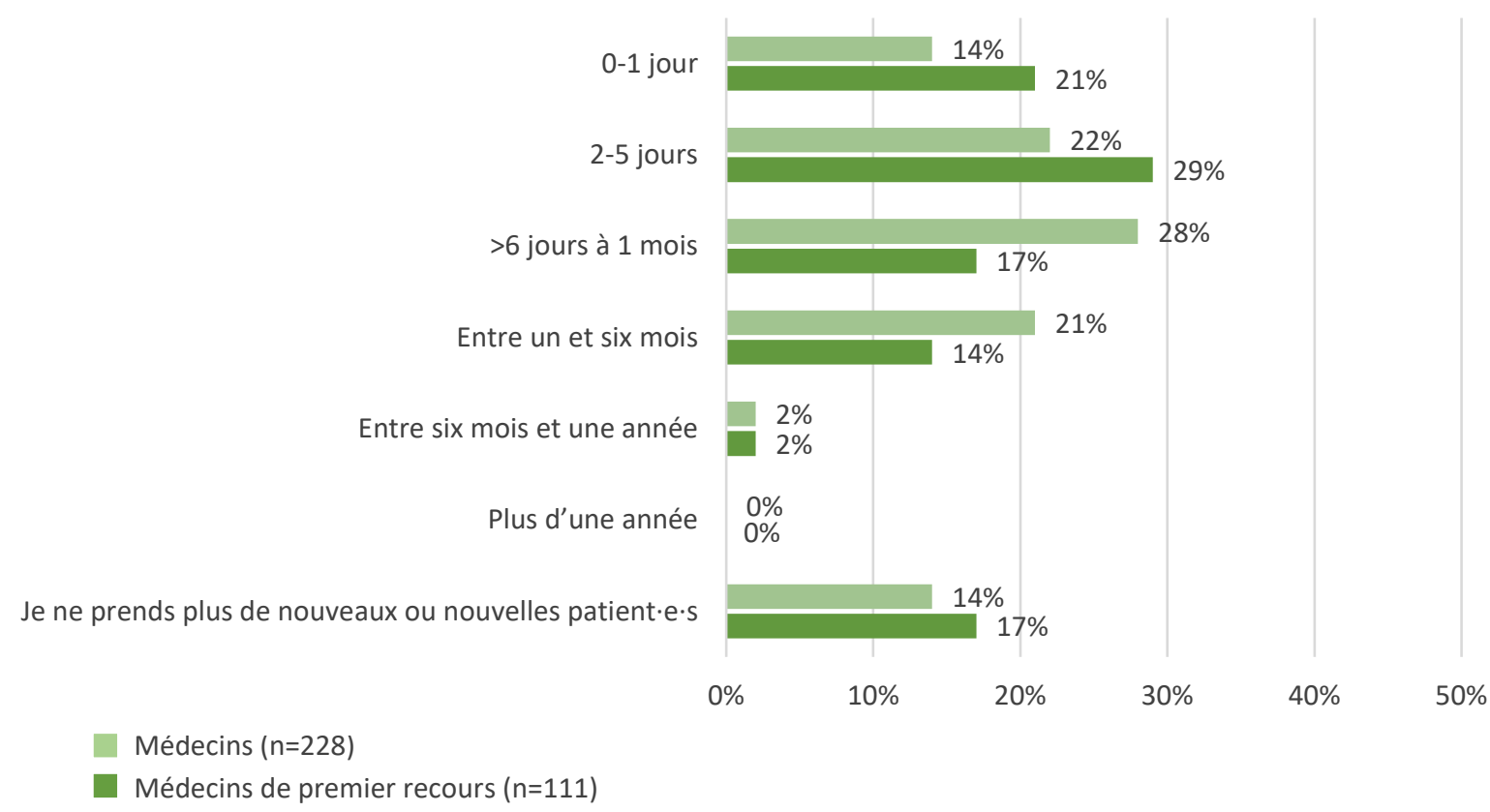

L'attente pour un nouveau ou une nouvelle patient e pour obtenir une consultation est de $0-5$ jours chez un tiers de l'ensemble des participant $\cdot e \cdot s$ et chez la moitié des participant $\cdot e \cdot s$ du sous-groupe des MPR. Dans les deux groupes, moins de $20 \%$ ne prend plus de nouveau ou nouvelle patient $\cdot e$. 


\section{Evaluation de l'offre médicale}

Tableau 7.1 Appréciation par les participant·e·s de l'offre médicale actuelle dans le canton pour leur propre spécialité.

\begin{tabular}{lcc}
\hline Offre médicale & $\mathbf{N}_{\text {total }}(\%)$ & $\mathbf{N}_{\text {MPR }}(\%)$ \\
\hline Insuffisante & $102(45)$ & $61(55)$ \\
\hline Suffisante & $98(43)$ & $39(35)$ \\
\hline Trop importante & $7(3)$ & $1(1)$ \\
\hline Sans avis & $21(9)$ & $10(9)$ \\
\hline Total & $\mathbf{2 2 8 ( 1 0 0 )}$ & $\mathbf{1 1 1 ( 1 0 0 )}$ \\
\hline
\end{tabular}

L'appréciation de l'ensemble des participant·ess est partagée entre une offre insuffisante et suffisante selon leur spécialité. Plus de la moitié des MPR trouve l'offre dans sa spécialité insuffisante dans le canton.

Tableau 7.2 Spécialités pour lesquelles les patient·e·s ont le plus de difficulté à obtenir un rendez-vous, selon les participant $\cdot e \cdot s$ (plusieurs réponses possible).

\begin{tabular}{|c|c|c|}
\hline Spécialités & $\mathbf{N}_{\text {total }}(\%)$ & $\mathrm{N}_{\text {MPR }}(\%)$ \\
\hline Dermatologie & $100(44)$ & $47(42)$ \\
\hline Neurologie & $98(43)$ & $67(60)$ \\
\hline Psychiatrie & $86(38)$ & $60(54)$ \\
\hline Rhumatologie & $75(33)$ & $58(52)$ \\
\hline Gastroentérologie & $48(21)$ & $28(25)$ \\
\hline Ophtalmologie & $45(20)$ & $15(14)$ \\
\hline Endocrinologie/Diabétologie & $43(19)$ & $24(22)$ \\
\hline Neurochirurgie & $43(19)$ & $33(30)$ \\
\hline Pneumologie & $25(11)$ & $17(15)$ \\
\hline Gynécologie/Obstétrique/Néonatologie & $24(11)$ & $14(13)$ \\
\hline Orthopédie & $22(10)$ & $16(14)$ \\
\hline Autre & $21(9)$ & $4(4)$ \\
\hline Gériatrie & $19(8)$ & $10(9)$ \\
\hline ORL & $15(7)$ & $5(5)$ \\
\hline Réadaptation & $15(7)$ & $9(8)$ \\
\hline Angiologie & $12(5)$ & $8(7)$ \\
\hline Cardiologie & $9(4)$ & $4(4)$ \\
\hline Urologie & $9(4)$ & $5(5)$ \\
\hline Médecine générale & $9(4)$ & $0(0)$ \\
\hline Chirurgie de la main & $6(3)$ & $4(4)$ \\
\hline Pédopsychiatrie & $5(2)$ & $2(2)$ \\
\hline Oncologie/radio-oncologie & $3(1)$ & $2(2)$ \\
\hline
\end{tabular}




\begin{tabular}{lll}
\hline Chirurgie viscérale & $2(1)$ & $2(2)$ \\
\hline Pédiatrie & $2(1)$ & $1(1)$ \\
\hline Total des répondant·e·s & $\mathbf{2 2 8}$ & $\mathbf{1 1 1}$ \\
\hline
\end{tabular}

La dermatologie, la neurologie, la psychiatrie et la rhumatologie sont considérées comme les spécialités pour lesquelles les patient'e.s ont le plus de difficultés à obtenir un rendez-vous, y compris par le sousgroupe des MPR.

Tableau 7.3 Appréciation par les participant·e·s de l'offre médicale DANS 5 ANS dans leur propre spécialité.

\begin{tabular}{lc}
\hline Offre & $\mathbf{N}_{\text {total }}(\%)$ \\
\hline Insuffisante & $99(43)$ \\
\hline Plutôt suffisante & $52(23)$ \\
\hline Suffisante & $46(20)$ \\
\hline Plus que suffisante & $13(6)$ \\
\hline Trop importante & $5(2)$ \\
\hline Sans avis & $13(6)$ \\
\hline Total & $\mathbf{2 2 8 ( 1 0 0 )}$ \\
\hline
\end{tabular}

Parmi les participant·e.s, environ $40 \%$ trouve que l'offre médicale dans sa spécialité sera insuffisante dans 5 ans.

Tableau 7.4 Appréciation par les participant·es de l'offre médicale DANS 5 ANS pour LA MEDECINE DE PREMIER RECOURS.

\begin{tabular}{lcc}
\hline Offre & $\mathbf{N}_{\text {total }}(\%)$ & $\mathbf{N}_{\text {MPR }}(\%)$ \\
\hline Insuffisante & $128(56)$ & $64(58)$ \\
\hline Plutôt suffisante & $51(22)$ & $29(26)$ \\
\hline Suffisante & $27(12)$ & $14(12)$ \\
\hline $\begin{array}{l}\text { Plus que } \\
\text { suffisante }\end{array}$ & $2(1)$ & $1(1)$ \\
\hline Trop importante & $1(1)$ & $1(1)$ \\
\hline Sans avis & $19(8)$ & $2(2)$ \\
\hline Total & $\mathbf{2 2 8 ( 1 0 0 )}$ & $\mathbf{1 1 1 ( 1 0 0 )}$ \\
\hline
\end{tabular}

Parmi l'ensemble des participant·es et dans le sous-groupe des MPR, plus de la moitié trouve que l'offre médicale pour la médecine de premier recours sera insuffisante dans 5 ans. 


\section{Discussion des résultats et conclusion}

Les résultats présentés dans ce rapport doivent être interprétés avec prudence, car le taux de réponse à l'enquête (22\%) est relativement bas. Diverses explications peuvent être avancées pour expliquer cette participation modeste, comme une surcharge de travail des médecins, possiblement exacerbée par la pandémie de COVID-19, ou les sollicitations régulières dont ils et elles font l'objet pour participer à des enquêtes.

Les résultats de la présente enquête sont toutefois intéressants et offrent un socle pour alimenter la réflexion sur la démographie médicale dans le canton de Fribourg, notamment en ce qui concerne la médecine de premier recours, puisque la moitié des participant.e.s (49\%) sont des MPR.

La plupart des participant-e.s sont des hommes, y compris dans le sous-groupe des MPR. Cette répartition se retrouve dans les statistiques médicales au niveau suisse ${ }^{1,2,3}$.

L'âge médian des participant·e.s est de 54 ans (48 ans chez les femmes et 57 ans chez les hommes). Les femmes sont plus nombreuses que les hommes dans la tranche d'âge des moins de 55 ans, ce qui pourrait indiquer une progressive féminisation de la profession, en particulier chez les MPR chez qui l'âge médian des femmes est de 45 ans (voir Tableau 1.1 dans la partie "Résultats détaillés »).

Le nombre de demi-journées moyennes d'activité hebdomadaire est un peu plus élevé

\footnotetext{
${ }^{1}$ Hostettler S, Kraft E. Statistique médicale 2020 de la $\mathrm{FMH}$ : la Suisse en comparaison internationale. Bulletin des médecins suisses. 2021;102(12):41722.

2 Stierli R, Rozsnyai Z, Felber R, Jorg R, Kraft E, Exadaktylos AK, et al. Primary Care Physician Workforce 2020 to 2025 - a cross-sectional study for the Canton of Bern. Swiss medical weekly. 2021;151.

${ }^{3}$ Gloor V, Chiolero A. Observatoire valaisan de la santé (OVS). Enquête sur l'activité des médecins en Valais. 2018. Sion, Mai 2019

4 Stierli R, Rozsnyai Z, Felber R, Jorg R, Kraft E, Exadaktylos AK, et al. Primary Care Physician Workforce 2020 to 2025 - a cross-sectional study for the Canton of Bern. Swiss medical weekly. 2021;151.
}

dans le sous-groupe des MPR (7.5) que lorsque l'on considère l'ensemble des participant.e.s (7.3). Pour les MPR, cette moyenne correspond à celle que l'on trouve dans les cantons de Berne et du Valais (7.6 demi-journées d'activité hebdomadaire en moyenne $)^{4,5}$. Lorsque l'on considère l'ensemble des participant.e.s à cette enquête, en revanche, la moyenne est plus basse que celle qu'on retrouve pour la Suisse dans les statistiques de la $\mathrm{FMH}^{6}$ (8.5 demijournées en moyenne).

Parmi l'ensemble des participant $\cdot \mathbf{e} \cdot \mathbf{s}$, un tiers envisage de diminuer son taux d'activité dans les 5 ans, y compris dans le sous-groupe des MPR (en majorité d'une à deux demi-journées par semaine). Lorsque l'on considère l'ensemble des participant.e.s, ce chiffre est plus élevé que ce qui a été constaté en Valais en 2018 dans une étude similaire $(27 \%$ de projet de diminution du taux d'activité). Les chiffres concernant les MPR sont, quant à eux similaires ${ }^{7}$. Chez les MPR, cette diminution concerne pour moitié les 65 ans et plus, ce qui s'explique avec des départs à la retraite. II serait toutefois nécessaire d'investiguer plus les raisons des médecins de moins de 60 ans qui envisagent de diminuer leur taux d'activité.

Un tiers de l'ensemble des participant $\cdot e \cdot s$, ainsi que la moitié du sous-groupe des MPR peut recevoir un nouveau ou une nouvelle patient $e$ dans un délai de 5 jours. De plus, $13 \%$ des médecins (17\% des MPR) déclare ne plus accepter de nouveaux patients ou de nouvelles patientes. Pour cette dernière donnée, malgré des chiffres un peu plus élevés qu'en Valais (12\% de médecins n'acceptant plus de nouveau ou nouvelle patient $\cdot e, y$ compris chez les MPR) ${ }^{8}$ le résultat reste un indicateur rassurant pour la

${ }^{5}$ Gloor V, Chiolero A. Observatoire valaisan de la santé (OVS). Enquête sur l'activité des médecins en Valais. 2018. Sion, Mai 2019

${ }^{6}$ Hostettler S, Kraft E. Statistique médicale 2020 de la $\mathrm{FMH}$ : la Suisse en comparaison internationale. Bulletin des médecins suisses. 2021;102(12):41722.

7 Gloor V, Chiolero A. Observatoire valaisan de la santé (OVS). Enquête sur l'activité des médecins en Valais. 2018. Sion, Mai 2019

8 Gloor V, Chiolero A. Observatoire valaisan de la santé (OVS). Enquête sur l'activité desmédecins en Valais. 2018. Sion, Mai 2019 
couverture actuelle de soins ambulatoires dans le canton de Fribourg.

\section{Conclusion et perspective}

En conclusion, sous réserve de la représentativité des résultats, cette enquête montre que l'âge médian des médecins du canton de Fribourg est de 54 ans, et de 50 ans pour les MPR. Sur la base du délai pour accepter un nouveau patient ou une nouvelle patiente au cabinet, l'offre actuelle semble suffisante, mais d'autres données sont nécessaires pour mieux l'évaluer. Par ailleurs, les MPR travaillent essentiellement dans des cabinets de groupe. Enfin, il existe une progressive féminisation de cette profession dans la mesure où les femmes sont plus nombreuses dans les classes d'âge les plus basses.
Toutefois, les prévisions à 5 ans sont plus préoccupantes si l'on tient compte de l'appréciation des médecins sur l'offre médicale à venir et de leur l'âge moyen. Enfin, même si le délai d'attente pour qu'un'e patient.e soit $\mathrm{vu} \cdot \mathrm{e}$ chez un'e MPR est relativement court, il est nécessaire d'envisager des possibilités de prise en charge pour les patient.e.s sans médecin traitant pour un suivi dès leur sortie de l'hôpital, ou pour une alternative à une consultation dans un services d'urgences pour les patient.e.s avec une urgence qui ne nécessite pas une consultation immédiate.

Un monitoring régulier de l'offre médicale dans le canton de Fribourg est nécessaire afin de suivre l'évolution de l'offre et des pratiques professionnelles. 


\section{Annexe 1 : Questionnaire}

Questionnaire sur l'activité des médecins

II y a 20 questions dans ce questionnaire, ce qui vous prendra environ 5 minutes.

\section{Partie 1 : Données administratives}

\subsection{Données socio-démographiques :}

Année de naissance :

Genre :

Féminin

Masculin

District :
○ Glâne
- Gruyère
- Veveyse
- Broye
- Sarine
- Singine
- Lac

\subsection{Structure du cabinet :}

Veuillez sélectionner parmi les propositions suivantes celle qui définit le mieux la structure principale dans laquelle vous travaillez:

Cabinet individuel

Cabinet à deux médecins ou de groupe

Médecin dans un établissement hospitalier

Autre :

1.3. Titre de spécialiste (titre ISFM ou reconnaissance MEBEKO) :

Veuillez choisir toutes les réponses qui conviennent :

Allergologie et immunologie clinique

Anesthésiologie

Angiologie

Cardiologie

Chirurgie

Chirurgie vasculaire

$\square$ Chirurgie cardiaque et vasculaire thoracique

$\square \quad$ Chirurgie de la main 
Chirurgie orale et maxillo-faciale

Chirurgie orthopédique et traumatologie de l'appareil locomoteur

Chirurgie pédiatrique

Chirurgie plastique, reconstructive et esthétique

Chirurgie thoracique

Dermatologie et vénéréologie

Endocrinologie-diabétologie

Gastroentérologie

Génétique médicale

Gériatrie

Gynécologie et obstétrique

Hématologie

Néphrologie

Infectiologie

Médecin praticien

Médecine du travail

Médecine interne générale

Médecine légale

Médecine nucléaire

Médecine pharmaceutique

Médecine physique et réadaptation

Médecine tropicale et médecine des voyages

Neurochirurgie

Neurologie

Neuropathologie

Oncologie médicale

Ophtalmologie

Oto-rhino-laryngologie

Pathologie

Pédiatrie

Pharmacologie et toxicologie cliniques

Pneumologie

Prévention et santé publique

Psychiatrie et psychothérapie

Psychiatrie et psychothérapie d'enfants et d'adolescents 


\section{Radiologie}

Radio-oncologie/radiothérapie

\section{Rhumatologie}

Soins intensifs

Urologie

Autre/ formation approfondie : 


\section{Partie 2 : Enquête sur l'activité du médecin}

Cette partie du questionnaire porte notamment sur votre type et degré d'activité, la qualité des soins et l'offre médicale ambulatoire.

\section{Activités}

\subsection{Principale activité - principale spécialisation exercée :}

Veuillez sélectionner une seule des propositions suivantes :

Allergologie et immunologie clinique

Anesthésiologie

Angiologie

Cardiologie

Chirurgie

Chirurgie vasculaire

Chirurgie cardiaque et vasculaire thoracique

Chirurgie de la main

Chirurgie orale et maxillo-faciale

$\square$ Chirurgie orthopédique et traumatologie de l'appareil locomoteur

$\checkmark$ Chirurgie pédiatrique

Chirurgie plastique, reconstructive et esthétique

- Chirurgie thoracique

Dermatologie et vénéréologie

$\checkmark$ Endocrinologie-diabétologie

Gastroentérologie

Génétique médicale

Gynécologie et obstétrique

Hématologie

Maladies des reins (Néphrologie)

Maladies infectieuses (Infectiologie)

Médecin praticien

Médecine du travail

Médecine interne générale

Médecine légale

Médecine nucléaire

Médecine pharmaceutique

Médecine physique et réadaptation 
Médecine tropicale et médecine des voyages

Neurochirurgie

Neurologie

Neuropathologie

Oncologie médicale

Ophtalmologie

Oto-rhino-laryngologie

Pathologie

Pédiatrie

Pharmacologie et toxicologie cliniques

Pneumologie

Prévention et santé publique

Psychiatrie et psychothérapie

Psychiatrie et psychothérapie d'enfants et d'adolescents

Radiologie

Radio-oncologie/radiothérapie

Rhumatologie

Soins intensifs

Urologie

Autre :

2.2. Combien d'heures par semaine en moyenne consacrez-vous à la pratique de votre métier, y compris l'administration ?

Entrée chiffrée

\subsection{Nombre de demi-journée(s) d'activité totale lors d'une semaine habituelle :}

Veuillez sélectionner une seule des propositions suivantes :
0
1
2
3
4
5
6
7 


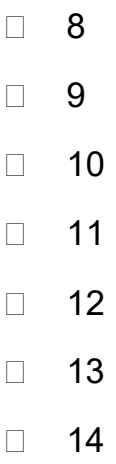

2.4. Pratiquez-vous la médecine de $1^{\text {er }}$ recours (médecine interne, médecine générale ou pédiatrie) ?

Veuillez sélectionner une seule réponse possible

$$
\text { Oui } \quad \square \text { Non }
$$

$\Rightarrow$ Si oui, question $n^{\circ} 2.5$

$\Rightarrow$ Si non, question $n^{\circ} 2.6$

2.5. Nombre de demi-journée(s) d'activité clinique de médecine de $1^{\text {er }}$ recours lors d'une semaine habituelle :

Veuillez sélectionner une seule des propositions suivantes :

Contrôle logique en fonction des réponses à la question $n^{\circ} 2.3$

2.6. En moyenne combien d'heures par semaine consacrez-vous au travail administratif (gestion de l'activité, comptabilité, rapports d'assurance, lettres, etc) ?

Entrée chiffrée

2.7. En moyenne combien d'heures par mois consacrez-vous à votre formation continue ?

Entrée chiffrée

2.8. Participez-vous à la garde ?

Veuillez sélectionner une seule des propositions suivantes :
Oui
Non

$\Rightarrow \mathrm{Si}$ oui, question $\mathrm{n}^{\circ} 2.8 .1$

$\Rightarrow$ Si non, question $n^{\circ} 2.9$

2.8.1. Si vous participez à la garde, combien de jours par année? De 0 à 365 jours 
2.9. Avez-vous le projet de modifier votre taux d'activité dans les cinq prochaines années : Veuillez sélectionner une seule des propositions suivantes :

Non

Oui, diminution

Oui, augmentation

$\Rightarrow$ Si oui, diminution : question $n^{\circ} 2.9 .1$

$\Rightarrow$ Si oui, augmentation : question $n^{\circ} 2.9 .2$

$\Rightarrow$ Si non, question $n^{\circ} 2.10$

2.9.1. Si oui, préciser la diminution planifiée en nombre de demi-journées par semaine : Veuillez sélectionner une seule des propositions suivantes :

1 à 2 demi-journées en moins

3 à 4 demi-journées en moins

5 demi-journées et plus en moins

2.9.2. Si oui, préciser l'augmentation planifiée en nombre de demi-journées par semaine : Veuillez sélectionner une seule des propositions suivantes :

Idem items question $n^{\circ} 2.9 .1$

\section{Evaluation de l'offre}

2.10. Quelle est la durée d'attente d'un nouveau patient pour obtenir un rendez-vous à votre consultation « normale » (urgence vitale exclue) ?

Veuillez sélectionner une seule des propositions suivantes :

0-1 jour

2-5 jours

$>6$ jours à 1 mois

Entre un et six mois

Entre six mois et une année

Plus d'une année

Je ne prends plus de nouveaux patients

2.11. Comment évaluez-vous l'offre médicale pour votre spécialité dans le canton de Fribourg?

Veuillez sélectionner une seule des propositions suivantes :

Insuffisante

Suffisante

Trop importante

Sans avis 
2.12. Quelles sont selon vous les spécialités pour lesquelles les patients ont plus de mal à trouver un rendez-vous?

Veuillez choisir toutes les réponses qui conviennent:

Cardiologie

Chirurgie viscérale

Dermatologie

Gynécologie/Obstétrique/Néonatologie

Ophtalmologie

ORL

Orthopédie

Rhumatologie

Réadaptation

Psychiatrie

Urologie

Endocrinologie/Diabétologie

Angiologie

Gastroentérologie

Pneumologie

Oncologie/radio-oncologie

Neurologie

Neurochirurgie

Gériatrie

Autre :

2.13. Quelle sera, à votre avis, l'offre dans votre spécialité dans le canton de Fribourg dans les 5 années à venir?

Veuillez sélectionner une seule des propositions suivantes :

Insuffisante

Plutôt suffisante

Suffisante

Plus que suffisante

Trop importante

Sans avis 
2.14. Quelle sera, à votre avis, l'offre pour la médecine de $1^{\text {er }}$ recours dans le canton de Fribourg dans les 5 années à venir ?

Veuillez sélectionner une seule des propositions suivantes :

$\square \quad$ Insuffisante

Plutôt suffisante

Suffisante

Plus que suffisante

Trop importante

- Sans avis

Merci d'avoir complété ce questionnaire. 